\title{
Can Enumerative Induction Be Defended by Modal Scepticism and Modal Empiricism?
}

\author{
SHUYI FENG Wuhan University
}

ABSTRACT: In the spirit of modal scepticism, Peter Hawke offers a modal epistemology, the safe explanation theory (SET), which takes the form of modal empiricism. By employing SET, he tries to defend enumerative induction (EI): it is reasonable to believe that any $X$ is $F$ on the basis of a sufficiently large sample in which any $X$ is $F$. In this paper, I argue that Hawke's defence fails. Moreover, I point out a problem with SET, which results in this failure: SET is too strict to account for some possibility claims that we are entitled to believe.

RÉSUMÉ : Dans l'esprit du scepticisme modal, Peter Hawke propose une épistémologie modale, la théorie de l'explication sûre (SET), qui adopte la forme de l'empirisme modal. Avec la SET, il essaie de défendre l'induction énumérative (EI) : on croit raisonnablement que tout X est $F$ sur la base d'un échantillon suffisamment grand dans lequel tout X est F. Dans cet article, je soutiens que l'argmentation de Hawke échoue. De plus, je signale un problème avec la SET, lequel cause cet échec: la SET est trop stricte pour expliquer certaines affirmations possibles que nous avons le droit de croire.

Keywords: modal epistemology, modal scepticism, modal empiricism, safe explanation theory, similarity principle, enumerative induction

\section{Introduction}

With respect to possibility, what we know and how we know are two central questions that philosophers working in the area of modal epistemology aim to

Dialogue 59 (2020), 345-361

(C) Canadian Philosophical Association/Association canadienne de philosophie 2019 doi:10.1017/S0012217319000350 
answer. ${ }^{1}$ As to the former, some endorse modal scepticism, which is the view that, while we have a good deal of knowledge of mundane possibility claims, our ability to establish exotic possibility claims is limited. As to the latter, there is a rationalist/empiricist dichotomy. Modal rationalists hold the view that we arrive at modal knowledge via a priori means, while modal empiricists maintain that our modal knowledge derives from empirical investigations.

Peter Hawke, a researcher at the Institute for Logic, Language and Computation at the University of Amsterdam, is both a modal sceptic and empiricist. In his 2011 paper, "Van Inwagen's Modal Skepticism," he provides a defence for Peter van Inwagen's modal scepticism. In the spirit of modal scepticism, in his 2017 paper, "Can Modal Skepticism Defeat Humean Skepticism?," he proposes a modal epistemology that takes the form of modal empiricism, i.e., the safe explanation theory (SET). Furthermore, he uses SET as a tool for defending enumerative induction (EI): on the basis of a sufficiently large sample in which any $X$ is $F$, and in the absence of any evidence that some $X$ is not $F$, it is reasonable to believe that any $X$ is $F{ }^{2}$ Hawke constructs an argument for EI and defends its second premise (P2) by employing SET.

In my paper, I have two aims. First, I aim to show that Hawke's defence for P2 fails. Second, I point out a problem with SET that leads to his failure of defence: SET is too strict to account for some possibility claims that we are entitled to believe. ${ }^{3}$ My plan for the remainder of this paper is as follows. In Section II, I clarify some basic notions and distinctions, as well as some representative positions in the area of modal epistemology. I also explain why Hawke dismisses modal rationalism and embraces modal empiricism: he believes that modal rationalism faces a dilemma. Section III is a sketch of Hawke's modal epistemology, i.e., SET. In Section IV, I present Hawke's argument for EI and rephrase how he defends P2. In Section V, I argue that his defence fails and I explain the cause of this failure. I respond to two possible objections and discuss a further question in Section VI. In the concluding Section VII, I show that SET, taking the form of modal empiricism, faces the same dilemma as modal rationalism.

1 In this paper, I put the difference between 'knowledge' and 'justification' aside and use the words interchangeably. Moreover, I use statements like 'we are entitled to believe that,' 'it is reasonable to believe that,' 'our belief in so and so is justified/ reasonable,' etc., interchangeably.

2 EI is a form of inductive inference that reasons from some members of a set to all members of the set. For example, if all swans observed are white, EI allows us to draw a universal conclusion that all swans are white.

3 Note that my position is weak. I do not intend to argue that P2 is false, or that Hawke's argument for EI is unsound. Rather, I merely aim to show that Hawke's defence for P2 is unsuccessful, which makes it an open question whether his argument for EI is sound. 


\section{Central Questions of Modal Epistemology}

The field of modal epistemology deals with our knowledge of what is possible (and what is necessary). The following are three important questions to consider: 1) what kind of possibility is under discussion?;2) which possibility claims are we entitled to believe?; 3) how do we arrive at possibility knowledge? Before moving on, a glance at the existing answers to these questions is necessary.

As to the first question about the types of possibilities under discussion, possibility abounds. Some kinds of possibility are epistemic (in other words, agent-dependent), and others are not. ${ }^{4}$ Among non-epistemic possibilities, metaphysical possibility, which is also called possibility 'simpliciter' or 'real' possibility, is widely accepted as primitive and "as the default reading of "it is possible that" or "things might have been that." 5 This metaphysical possibility is the central focus not only of the field of modal epistemology, but likewise of Hawke's paper and of this paper. ${ }^{6}$

Besides metaphysical possibility, two other non-epistemic possibilities, namely, (broad) logical possibility and (broad) physical possibility, are also commonly found in philosophical discussions. As is generally accepted, $p$ is logically possible if and only if there is no (logical, mathematical, semantical, conceptual, etc.) contradiction deduced from $p ; p$ is physically possible if and only if $p$ is compatible with the laws of nature. Given how metaphysical possibility is characterized $-p$ is metaphysically possible if and only if $p$ is compatible with the natures of things that could have existed - there is a standard understanding of the relation between these possibilities. Metaphysical possibility is less expansive than logical possibility: it is logically but not metaphysically possible that Barbara Bush is Barack Obama's mother. Metaphysical possibility is more expansive than physical possibility: it is metaphysically but not physically possible that the velocity of something is more than the speed of light. Accordingly, metaphysical necessity is less expansive than physical necessity: it is physically but not metaphysically necessary that nothing travels faster than light. This standard understanding of the relations between these kinds of possibilities/necessities, especially the relation between metaphysical possibility/necessity and physical possibility/ necessity, will be presupposed as a working premise in this paper. ${ }^{7}$

The distinction between necessity and regularity should also be considered. Take the maximum height of a high jump as an example. The biological structure

4 Gendler and Hawthorne provide a detailed summary of the characterizations of epistemic possibility. See Gendler and Hawthorne (2002), p. 3.

5 Hawke (2017), p. 286. See also van Inwagen (1998), Gendler and Hawthorne (2002), and Vaidya (2017) for this standard understanding of metaphysical possibility.

6 For the sake of brevity, the words 'possibility/necessity' in the following refer to metaphysical possibility/necessity if there is no further qualification.

7 About the standard understanding of the relations between different kinds of possibilities/necessities, see Gendler and Hawthorne (2002), p. 5, and Vaidya (2017). To be sure, other philosophers have different views. For example, Bealer (2002) 
of human beings and gravity, together with some other biomechanical factors, determine the maximum height of a high jump, say, a certain height less than 6 metres. Thus, that no human being can jump 6 metres high is a physical necessity. In contrast, that no human being can jump 2.46 metres high is probably not a physical necessity, but a mere accidental general fact. The current world record for the men's high jump is 2.45 metres, which is expected to be broken. Given the distinction between physical and metaphysical necessity, we can go one step further by concluding that it is not metaphysically necessary that no human being can jump 2.46 metres high.

As to the second question about which types of possibility claims we are entitled to believe, there is no doubt that we do have knowledge of some possibility claims (although it is controversial from where that knowledge is derived). Consider two mundane possibility claims: "it is possible that "the table that was in a certain position at noon have then been two feet to the left of where it in fact was"" and "it is possible that "John F. Kennedy have died of natural causes." 8 These claims are intuitive and face no defeaters, which lends some support to the view that we are entitled to believe them. In contrast, with regard to some more exotic possibility claims, such as "a (naturally) purple cow is possible" or "transparent iron is possible," it seems we are not entitled to believe them, or to say the least, it is not clear whether we are entitled to believe them. ${ }^{9}$ Modal sceptics hold that, while we know many mundane possibility claims, the inquiry of exotic possibility knowledge is out of our reach. Thus, if a modal epistemology were able to establish an exotic possibility claim, then a modal sceptic would be suspicious of accepting this epistemology.

As to the third question about how we arrive at possibility knowledge, there is a rationalist/empiricist dichotomy. Modal rationalism, which is the view that modal knowledge derives in an a priori way, has been dominant for the last few decades. Stephen Yablo and David Chalmers, as proponents of this view, endorse a so-called 'conceivability-possibility' principle (CP), which says that conceivability provides us with a guide to possibility. ${ }^{10}$ The core idea of CP is that if we can conceive of what a statement $\varphi$ describes (via our imaginative abilities), in the sense that we can construct a fictional story (i.e., a set of statements) that logically implies $\varphi$, then $\diamond \varphi$ is justified.

regards logical and metaphysical possibility as the same thing. Shoemaker (1998) and Bird (2005) equate metaphysical necessity with physical necessity. However, these views are not in accordance with Hawke's, given that he embraces the standard understanding. For his view on the relations between logical, metaphysical, and physical possibility, see Hawke (2017), pp. 286-287.

8 Van Inwagen (1998), p. 70.

9 Van Inwagen (1998), p. 78, 80.

10 More precisely, Chalmers endorses the entailment account, which says that conceivability entails possibility. Yablo advocates a weaker view, the evidential account, which says that conceivability only provides evidence of possibility. See Chalmers (2002), Yablo (1993). 
Hawke is dissatisfied with $\mathrm{CP}$ because he finds that $\mathrm{CP}$ faces a dilemma: when we do a conceivability exercise, either we do or we do not pose some constraints on the story we produce. If the story is unconstrained, in the sense that it incorporates claims whose possibilities have not already been justified, then $\mathrm{CP}$ can provide a justification for exotic or even false possibility claims. For example, we are able, with ease, to construct stories in which the following claims are true: (naturally) purple cows exist; water is not $\mathrm{H}_{2} \mathrm{O}$; Goldbach's conjecture is false, etc. ${ }^{11}$ By employing the unconstrained $\mathrm{CP}$, we can conclude that they are possible, which is a result that we are reluctant to accept, and which therefore casts doubt on the unconstrained $\mathrm{CP}$.

Hawke instead proposes a conservative version of $\mathrm{CP}$, which requires each statement $\varphi_{1}, \varphi_{2}, \ldots, \varphi_{\mathrm{n}}$ incorporated in a fictional story be "modally safe," in the sense that each accompanying possibility statement, i.e., $\diamond \varphi_{1}, \diamond \varphi_{2}, \ldots, \diamond \varphi_{\mathrm{n}}$ has already been justified. ${ }^{12}$ In other words, the conservative $\mathrm{CP}$ requires that the justification of a possibility claim in question derive from claims whose possibilities are already justified. Thus, claims that are not modally safe, such as 'water is not $\mathrm{H}_{2} \mathrm{O}$,' 'Goldbach's conjecture is false,' and '(naturally) purple cows exist,' are not qualified to constitute stories. As a result, the conservative $\mathrm{CP}$ avoids establishing exotic or even false possibility claims.

However, as Hawke claims, the conservative CP is too strict to account for some possibility claims that we are entitled to believe. The conservative $\mathrm{CP}$ accounts for possibility claims in terms of other justified possibility claims, which, however, may result in a regress. To avoid the possible regress, justification must be halted somewhere. According to Hawke, there are a set of foundational claims, which are modally safe and therefore qualified to constitute a story, but whose possibilities are not justified by constructing a story. In other words, it is some other methods, rather than the conservative $\mathrm{CP}$, that justify the possibilities of the foundational claims. ${ }^{13}$

In sum, CP faces a dilemma: the unconstrained CP is so liberal that it establishes some possibility claims that we are not entitled to believe; the conservative $\mathrm{CP}$ is too strict to account for some possibility claims that we are entitled to believe. This dilemma motivates Hawke to abandon modal rationalism and embrace modal empiricism, which is the view that our modal knowledge derives from empirical investigations. In the following section, I will outline the main idea of the modal epistemology he proposes, i.e., SET.

11 Goldbach's conjecture is a conjecture in number theory, which says that every even number that is greater than 2 is the sum of two primes. In this paper, I assume that Goldbach's conjecture is true.

12 Hawke (2017), p. 294.

13 Hawke suggests that the possibilities of the foundational claims "are justified by the actuality principle and the similarity principle." See Hawke (2011), p. 360. For more about the actuality principle and the similarity principle, see also Hawke (2017), Roca-Royes (2017), and Section III of my paper. 


\section{A Sketch of Hawke's Modal Epistemology}

A satisfactory modal epistemology should avoid establishing exotic or false possibility claims, which we are not entitled to believe. If a modal epistemology allowed us to conclude that '(naturally) purple cows possibly exist,' "transparent iron is possible,' or 'it is possible that Barbara Bush is Barack Obama's mother,' then we had better deny it. Due to this consideration, Hawke is committed to (only) the following four empirically informed methods, which together constitute SET:

1. Actuality Principle: If it is reasonable to believe that $\varphi$, it is reasonable to believe that $\varphi$ is possible. ${ }^{14}$

2. Abduction Principle: If part of the best explanation for established fact $\varphi$ is that $\psi$ is possible (or necessary), then it is reasonable to believe that $\psi$ is possible (or necessary). ${ }^{15}$

3. Similarity Principle: If it is reasonable to believe that $F$ is a possible property for object $o_{1}$ and $o_{2}$ appears relevantly similar to $o_{1}$, then it is reasonable to believe that $F$ is a possible property of $o_{2} \cdot{ }^{16}$

4. Modest Recombination: If it is reasonable to believe that $\varphi$ is possible, that $\psi$ is possible, and that $\varphi$ and $\psi$ describe independently existing states of affairs, then it is reasonable to believe that $\varphi \wedge \psi$ is possible. ${ }^{17}$

Some other methods, although being empirically informed, are dismissed in the first place. Take property recombination as an example:

Property Recombination: If it is reasonable to believe that object $o$ could exist and that property $F$ could be instantiated, then it is reasonable to believe that $o$ could be $F .18$

For one thing, property recombination would establish some exotic possibilities, such as the possibility of purple cows or transparent iron. For another, it justifies false possibility claims. Here is the example Hawke offers: Ann Dunham is

14 Hawke (2017), p. 296.

15 Ibid.

16 Ibid. Moreover, Hawke is not explicit on how to understand the relation of relevantly similar counterparts, which I will discuss in Sections IV, V, and VI.

17 In his paper, Hawke in fact writes: "it is reasonable to believe that $\varphi \wedge \psi, \varphi \wedge \neg \psi$, $\neg \varphi \wedge \psi$ and $\neg \varphi \wedge \neg \psi$ are all possible." See Hawke (2017), p. 296. However, there are cases in which our belief in $\diamond(\varphi \wedge \neg \psi), \diamond(\neg \varphi \wedge \psi)$ or $\diamond(\neg \varphi \wedge \neg \psi)$ is not reasonable. Here is an example: let $\varphi$ be 'water $=\mathrm{H}_{2} \mathrm{O}$;' let $\psi$ be 'Phosphorus $=$ Hesperus.' It is reasonable to believe that $\varphi$ is possible, that $\psi$ is possible, and that $\varphi$ and $\psi$ describe independently existing states of affairs. However, it is not reasonable to believe that any of $\varphi \wedge \neg \psi, \neg \varphi \wedge \psi$, and $\neg \varphi \wedge \neg \psi$ is possible. In order to make modest recombination more plausible, I drop ' $\varphi \wedge \neg \psi, \neg \varphi \wedge \psi$ and $\neg \varphi \wedge \neg \psi$ ' in my paper. Ibid. 
Barack Obama's mother and is therefore necessarily his mother. So it is impossible for someone else — Barbara Bush, for example — to be his mother. However, according to property recombination, given that Barbara Bush exists (and therefore possibly exists) and that the property of being Barack Obama's mother is instantiated (and therefore is possibly instantiated), it follows that we are entitled to believe that Barack Obama's mother could have been Barbara Bush. ${ }^{19}$ For these two reasons, property recombination is not qualified as a member of SET.

The above is a sketch of SET. Hawke employs SET as a tool to defend EI. In the next section, I will present his argument for EI and examine how he justifies P2 of this argument.

\section{Hawke's Defence of P2}

Consider the following case: we have observed a great number of emeralds, all of which are green. That is to say, we have no empirical evidence of non-green emeralds. In this case, are we entitled to believe that all emeralds, whether observed or unobserved, are green? If one were tempted to answer 'yes,' then one would embrace EI:

EI: On the basis of a sufficiently large sample in which any $X$ is $F$, and in the absence of any evidence that some $X$ is not $F$, it is reasonable to believe that any $X$ is $F .^{20}$

On the basis of the emerald case, Hawke proposes an argument for EI and provides the following formalization ${ }^{21}$ :

19 Hawke (2017), p. 297.

20 See Hawke (2017), p. 284. There are actually two versions of EI: the universal inference, which is an inference from $X \mathrm{~s}$ observed to all $X \mathrm{~s}$, and the predicative inference, which is an inference from $X$ s observed to the next $X$ to be observed. Hawke, however, claims that it does no harm to his argument if the two versions are regarded as equivalent. To simplify the discussion of my paper, I only discuss the universal inference.

21 The formalization is based on Hawke's original argument, which is:

"P1. S is reasonable to believe that green emeralds are possible.

$\mathrm{P} 2$. $\mathrm{S}$ is not reasonable to believe that yellow emeralds are possible.

P3. What an agent reasonably believes (given her empirical evidence) is a function of only those propositions that she reasonably considers to be possible.

C.Therefore: $\mathrm{S}$ is both reasonable to believe that the next emerald she encounters will be green and that every emerald is green." See Hawke (2017), p. 285.

One problem with this original argument is worth mentioning: as Hawke himself concedes, $\mathrm{P} 3$ is vague. This vagueness in turn makes it difficult to evaluate the validity of the argument as such. This is why I base my discussion on the formalized version of his argument rather than the original version. 
Let emerald $(x)$ stand for ' $x$ is an emerald' and green $(x)$ stand for ' $x$ is green,'22

$\mathrm{P} 1 . \mathrm{B}(\diamond \exists x(\operatorname{emerald}(x) \wedge \operatorname{green}(x)))$

P2. $\neg \mathrm{B}(\diamond \exists x($ emerald $(x) \wedge \neg$ green $(x)))$

P3. $\mathrm{B}(\diamond \exists x(\varphi(x) \wedge \psi(x))) \wedge \neg \mathrm{B}(\diamond \exists x(\varphi(x) \wedge \neg \psi(x))) \rightarrow \mathrm{B}(\forall x(\varphi(x) \rightarrow \psi(x)))$ for any $\varphi(x)$ and $\psi(x)$

C. $\mathrm{B}(\forall x(\operatorname{emerald}(x) \rightarrow \operatorname{green}(x)))^{23}$

P1 says that it is reasonable to believe in the possibility that some emeralds are green. P2 says that it is not reasonable to believe in the possibility that some emeralds are not green. Both are assertions about our justification status of a modal claim. P3 is an inference from our justification status of modal claims to that of a non-modal claim. This argument is valid. Its soundness is yet to be determined. In this paper, I will set aside how Hawke defends P1 and P3, and will concentrate on P2 only. I will first explain how he defends P2 and then go on to argue against his defence in the section following.

Hawke vindicates P2 by invoking SET. He examines each of the four methods that SET incorporates and argues that none of them can establish the possibility of non-green emeralds. It is easy to see that the actuality principle and the abduction principle fail to undertake this task: given that non-green emeralds have never been observed, the actuality principle is not applicable. Nor is the abduction principle, since it is not clear why positing the possibility of non-green emeralds helps to explain the fact that all emeralds observed are green.

Now we consider the similarity principle and modest recombination. The similarity principle applies only if it is reasonable to believe that a relevantly similar counterpart of emeralds observed could be not green. What kind of things can be regarded as relevantly similar counterparts of emeralds observed? Hawke does not offer an explicit answer. Nevertheless, the following passage gives us a clue:

Since the only relevant evidence is an observation stream of green emeralds, the agent does not have available evidence of relevantly similar objects that could be yellow, so the similarity principle is not applicable. ${ }^{24}$

In this passage, it seems that Hawke implicitly presupposes the following criterion concerning the counterpart relation: for any emerald, only another emerald can count as its relevantly similar counterpart. Given this presupposition, in order to apply the similarity principle, evidence for the possibility of non-green emeralds is required. However, the only tools available to us are the

22 In addition, B stands for 'it is reasonable to believe that'; while $\varphi$ and $\psi$ stand for any two properties.

23 Hawke (2017), p. 299.

24 Hawke (2017), p. 297. 
actuality principle and the abduction principle, neither of which can provide us with the evidence required. Thus, the possibility of non-green emeralds cannot be established by invoking the similarity principle. ${ }^{25}$

Modest recombination fails to justify the possibility of non-green emeralds, too. For one thing, modest recombination only applies to statements taking the form of a conjunction, but the statement 'some emerald is not green' is not a conjunction. For another, as Hawke argues, modest recombination does not allow us to establish the possibility of non-green emeralds merely on the basis of evidence that emeralds exist (and therefore possibly exist) and that the property of being non-green is instantiated (and therefore is possibly instantiated). It is property recombination that is able to do the work of this sort, but this was dismissed already at the outset. In sum, the possibility of non-green emeralds cannot be established via any method that SET subsumes. Given that SET is the only modal epistemology that Hawke accepts, he concludes that it is not reasonable to believe in the possibility of non-green emeralds.

\section{Hawke's Failure of Defence}

In this section, I will argue that Hawke's defence for P2 is untenable. I will explain this with the help of the following two example claims:

(C1) It is possible that some human being lives 150 years.

(C2) It is possible that some lump of pure gold weighs $10,000 \mathrm{~kg}$.

(C1) and (C2) are intuitive and confront no obvious defeaters, which makes our belief in $(\mathrm{C} 1)$ and $(\mathrm{C} 2)$ reasonable to the same extent as that in other mundane possibility claims, such as 'Kennedy could have died of natural causes.' Thus, the following claim about our justification status of $(\mathrm{C} 1)$ and $(\mathrm{C} 2)$ is plausible:

(J) It is reasonable to believe (C1) and (C2).

However, if we adopted the strategy that Hawke uses to defend P2, we would deny (J), which is an unacceptable result. In view of this, it follows that Hawke's defence for P2 is likewise unacceptable. Here is my argument against his defence:

$\mathrm{P} 4$. If $\mathrm{P} 2$ is acceptable for the reason that Hawke provides, then $(\mathrm{J})$ is unacceptable for the same reason.

P5. ( $\mathrm{J})$ is acceptable.

Conclusion: P2 is unacceptable for the reason that Hawke provides.

25 However, if we had evidence for the possibility of non-green emeralds by invoking the actuality principle or the abduction principle, then the similarity principle would be redundant in the emerald case. The redundancy makes Hawke's understanding of the counterpart relation dubious. I will consider this in Section V. 
This argument is valid. In the following, I will explain P4 and P5 in order. To vindicate P4 is to show that SET fails to account for (C1) and (C2). First, let's consider (C1). It is easy to see that the actuality principle does not apply, since 150-year-old human beings have never been observed.

Second, the abduction principle is not applicable, either, given that it is not clear how positing the possibility of 150-year-old human beings helps to explain the fact that no human being observed has lived up to 150 years.

Third, let's consider the similarity principle, which applies only if we are entitled to believe that a relevantly similar counterpart of human beings observed could have lived 150 years. In view of how Hawke understands the counterpart relation, only human beings can count as similar counterparts of other human beings. Thus, if the similarity principle applied, we would be required to have evidence for the very possibility of 150-year-old human beings. Since this requirement is not met, the similarity principle cannot be deployed.

Fourth, let's consider modest recombination. Given that the claim 'some human being lives 150 years' is not a conjunction, modest recombination cannot justify its possibility. Thus, no method in SET is able to account for (C1). Property recombination seems promising because it allows us to establish (C1) simply on the basis that human beings exist (and therefore possibly exist) and that the property of being 150-year-old is instantiated (and therefore is possibly instantiated). However, this method has been dismissed from the outset and is not incorporated in SET.

Likewise, for four reasons, it is easy to see that SET fails to account for (C2): first, given that a $10,000 \mathrm{~kg}$ lump of pure gold has never been observed, the actuality principle does not apply.

Second, the abduction principle is not applicable either, since it is not clear how positing the possibility of a $10,000 \mathrm{~kg}$ lump of pure gold helps to explain the fact that any lump of pure gold observed weighs less than $10,000 \mathrm{~kg}$.

Third, the similarity principle applies only if we are entitled to believe that a relevantly similar counterpart of lumps of pure gold observed possibly weighs $10,000 \mathrm{~kg}$. According to how Hawke understands the counterpart relation, only lumps of pure gold can count as similar counterparts of other lumps of pure gold. That is to say, that the similarity principle applies requires evidence for the very possibility that some lump of pure gold has a mass of $10,000 \mathrm{~kg}$. Since this requirement is not met, the similarity principle cannot be deployed.

Fourth, given that the claim 'some lump of pure gold weighs $10,000 \mathrm{~kg}$ ' is not a conjunction, modest recombination cannot justify its possibility. Thus, all methods in SET fail to account for (C2). Although property recombination allows us to establish (C2) simply based on the evidence that lumps of pure gold exist (and therefore possibly exist) and the property of weighing 10,000 kg is instantiated (and therefore is possibly instantiated), this method has been dismissed from the start and is not included in SET.

Thus, neither (C1) nor (C2) can be established by invoking SET. Since SET is the only modal epistemology available, we must deny that it is reasonable to 
believe $(\mathrm{C} 1)$ and $(\mathrm{C} 2)$, the same way Hawke denies that it is reasonable to believe in the possibility of non-green emeralds. That is to say, we must deny $(\mathrm{J})$ for the same reason that Hawke gives to endorse P2. This is why P4 holds.

Now, let's consider P5, which claims that $(\mathrm{J})$ is acceptable. A consideration of the following question will help us to see why $(\mathrm{J})$ is plausible:

For some ordered pair $\langle X, F\rangle$ such that any $X$, whether observed or unobserved, is $F$, is it reasonable to believe that it is possible that some $X$ is not $F$ ?

I suggest that there are (at least) two cases in which there is room to give a positive answer to this question:

(i) That any $X$ is $F$ is physically necessary.

(ii) That any $X$ is $F$ is a mere general fact.

In case (i) or (ii) holds, then all $X \mathrm{~s}$, whether observed or unobserved, are $F_{\mathrm{s}}$. However, from the fact that (i) or (ii) is the case, it does not follow that any $X$ 's being $F$ is metaphysically necessary. Given the distinction between metaphysical necessity, physical necessity, and generality, as is clarified in Section II, there is room for (i') or (ii') to be the case:

(i') That any $X$ is $F$ is physically necessary without being metaphysically necessary.

(ii') That any $X$ is $F$ is a mere general fact without being metaphysically necessary.

If (i') or (ii') is the case, then it is metaphysically possible that some $X$ is not $F$. Moreover, if we are entitled to accept the distinction between metaphysical necessity, physical necessity, and generality, then we are entitled to believe in the metaphysical possibility that some $X$ is not $F$. Thus, if (i') or (ii') is the case, we can give a positive answer to the question above. I will explain this with examples.

(i'): For some ordered pair $\langle X, F\rangle$, that any $X$ is $F$ is physically necessary without being metaphysically necessary. For example, that nothing travels faster than light is physically necessary. However, how fast an object can travel is necessitated by the laws of nature. It is nevertheless metaphysically possible that something travels faster than light. If it is reasonable to accept the distinction between physical and metaphysical necessity with respect to the speed of things, then it is reasonable to believe that something travelling faster than light is metaphysically possible.

In a similar vein, it is physically necessary that there is a limit to the human lifespan, say, 150 years. However, the maximum lifespan of human beings is biologically necessitated by the human genome and some environmental factors. It is nevertheless metaphysically possible that some human being breaks the 
ceiling of human maximum lifespan. Therefore, if we are entitled to accept the difference between physical and metaphysical necessity, then we are entitled to believe (C1).

(ii'): For some ordered pair $\langle X, F\rangle$, that any $X$ is $F$ is a mere general fact without being a necessity (in any sense). For example, it is a true generalization that there is no lump of pure gold that has a mass of $10,000 \mathrm{~kg}$. But this truth is accidental: we could make a lump of pure gold with a mass bigger than $10,000 \mathrm{~kg}$ if we chose to do so. Thus, if it is reasonable to accept the difference between a mere general fact and a metaphysical necessity, then it is reasonable to believe (C2).

Thus, $(\mathrm{C} 1)$ and $(\mathrm{C} 2)$ are true and therefore $(\mathrm{J})$ and P5 are true. Given that P4 and P5 are true, my argument against P2 is sound and Hawke's defence of P2 fails, which further leads to the failure of his defence of EI. In the following, I will go on to demonstrate the cause of this failure: SET is too strict to account for some possibility claims that we are entitled to believe.

As I have argued, for some ordered pair $\langle X, F\rangle$ such that (i) or (ii) holds, possibility claims with the form 'it is possible that some $X$ is not $F$ ' are true. Among these claims, at least for some (although not for all) of them, we are entitled to believe in their truths. (C1) and (C2) are two examples for this. However, for any ordered pair $\langle X, F>$ such that (i) or (ii) holds, we cannot have any evidence that some $X$ is not $F$. In this case, we cannot establish the possibility that some $X$ is not $F$ via SET. Thus, for some possibility claims with the form 'it is possible that some $X$ is not $F$, although we are justified in believing them, our justification cannot come from SET. I will elaborate on this point in the following.

First, our justification cannot derive from the actuality principle, whose application requires our evidence that some $X$ is $F$. Second, it cannot come from the abduction principle either. As Hawke argues, it is not clear how positing the possibility that some $X$ is not $F$ helps to explain the fact that any $X$ observed is $F$. Third, modest recombination applies to conjunctions only, but claims with the form 'some $X$ is not $F$ ' are not conjunctions. Fourth, Hawke's understanding of the counterpart relation renders the similarity principle inapplicable and redundant. ${ }^{26}$ In the emerald case, he takes an emerald as the counterpart of another emerald. Following this understanding, it seems that for any ordered pair $\langle X, F\rangle$, only an $X$ can be taken as standing in a counterpart relation to another $X$. That is to say, the similarity principle applies if we have already had evidence (perhaps via other methods) for the very possibility that some $X$ is not $F$. Since neither the actuality principle nor the abduction principle or modest recombination can provide us with such evidence, the similarity principle is rendered inapplicable. However, if we had evidence of the possibility that some $X$ is not $F$, the similarity principle would not be needed.

26 Hartl (2016) and Prelević (2015) hold similar views. Hartl maintains that the actuality principle has a very narrow explanatory scope. See Hartl (2016), p. 287. Prelević argues that the actuality principle is redundant. See Prelević (2015), p. 495. 


\section{Two Objections, Responses, and a Further Question}

In this section, I respond to two possible objections and then discuss a further question.

Objection to P5: We are not entitled to believe (C1) or (C2). That is to say, (J) and P5 are false.

My Response: First, the distinction between metaphysical necessity, physical necessity, and generality lends sufficient plausibility to the view that our belief in $(\mathrm{C} 1)$ and $(\mathrm{C} 2)$ is reasonable. I can think of no reason with which to refute this view. Perhaps my opponent, although she denies that our belief in $(\mathrm{C} 1)$ and $(\mathrm{C} 2)$ is reasonable, would like to acknowledge that our belief in some of the following claims is justified:

(C3) It is possible that some particle travels faster than the speed of light.

(C4) It is possible that some apple has a free-fall acceleration far more than $9.8 \mathrm{~m} / \mathrm{s}^{2}$.

(C5) It is possible that some human being can jump 6 metres high.

(C6) It is possible that some human being can jump 2.46 metres high.

(C7) It is possible that some woman is president of the United States.

If so, I would like to replace $(\mathrm{C} 1)$ and $(\mathrm{C} 2)$ in $(\mathrm{J})$ with any claim of the above. My argument against Hawke nevertheless remains intact even if I do so. If my opponent insists that none of the claims I list above are justified, I can offer more claims of the form 'it is possible that some $X$ is not $F$ ', for which $\mathrm{I}$ think there is justification. Second, I consider an extreme case in which — for any possibility claim I provide - my opponent denies that we have justification for it. Under this extreme circumstance, I would like to retreat to a more modest position:

( $\left.\mathrm{J}^{*}\right)$ For some ordered pair $\langle X, F\rangle$ such that any $X$ is $F$, it is reasonable to believe that it is possible that some $X$ is not $F$.

Compare $\left(\mathrm{J}^{*}\right)$ with $(\mathrm{J})$ :

(J) It is reasonable to believe (C1) and (C2).

$(\mathrm{J})$ is about some specific possibility claims, while $\left(\mathrm{J}^{*}\right)$ is a mere existential claim, which is about an unspecific possibility claim and which is weaker than $(\mathrm{J})$. By replacing $(\mathrm{J})$ with $\left(\mathrm{J}^{*}\right)$, my argument against Hawke, which takes a weaker form, runs as follows:

$\mathrm{P} 4 *$. If $\mathrm{P} 2$ is acceptable for the reason that Hawke provides, then $\left(\mathrm{J}^{*}\right)$ is unacceptable for the same reason.

P5*. ( $\left.{ }^{*}\right)$ is acceptable.

Conclusion: P2 is unacceptable for the reason that Hawke provides. 
The distinction between metaphysical necessity, physical necessity, and generality provides us with a good reason to accept $\left(\mathrm{J}^{*}\right)$. So P5*, which is weaker than P5 and which therefore faces a lower burden of proof, is plausible. Section V, in which I argue that SET's explanatory scope is too narrow, actually explains why P $5 *$ is true: following the way that Hawke defends P2, we must deny $\left(\mathrm{J}^{*}\right)$. So, even if I replace $(\mathrm{J})$ with $\left(\mathrm{J}^{*}\right)$ in my original argument, we can draw the same conclusion that Hawke's defence for P2 is unsuccessful.

Objection to P4: The antecedent of P4 is true without the consequence being true, which makes P4 false. SET's inability to account for (C1) and (C2) is rooted in the fact that we misunderstand the application condition of the similarity principle; in other words, our understanding of the counterpart relation is too narrow. In the case of $(\mathrm{C} 1)$, only human beings are regarded as qualified to be similar counterparts of other human beings. In the case of (C2), only lumps of pure gold are regarded as qualified to play the role of similar counterpart. However, the counterpart relation should be interpreted in a more liberal way. That is to say, the similar counterpart of a human being is not necessarily another human being. It could be a primate, a mammal, or any animal. If we take a 150-year-old turtle as the similar counterpart of human beings, then we can justify the possibility of a 150 -year-old human being by deploying the similarity principle. Similarly, if we take a $10,000 \mathrm{~kg}$ lump of stone as a similar counterpart of lumps of pure gold, then we can also justify the possibility of a 10,000 kg lump of pure gold. Furthermore, in view of this more liberal interpretation of the counterpart relation, the explanatory scope of SET will be expanded and therefore the criticism that SET is too strict to account for some justified possibility claims can be refuted.

My Response: I quite agree that it is not necessary to read the counterpart relation in as narrowly as Hawke does. Sonia Roca-Royes offers a detailed clarification on the application of the similarity principle, especially on the counterpart relation with the following example: it seems a justified belief that her Ikea pine-wood table, Messy, could have been broken. How does this justification derive? First, she knows that Messy's similar counterpart, Twin-Messy, actually breaks. Then, by deploying the similarity principle, she can infer that Messy could have been broken. Now a natural question poses itself: which kind of thing can count as Messy's similar counterpart? Another Ikea pine-wood table? A pine-wood table? Or any wooden table? As Roca-Royes argues, any of them is qualified to play the role of counterpart. ${ }^{27}$

Following this liberal interpretation of the counterpart relation, to be sure, as my opponent claims, $(\mathrm{C} 1)$ and $(\mathrm{C} 2)$ can be justified via the similarity principle, which makes the consequence of $\mathrm{P} 4$ false. However, it should be noted that the liberal interpretation also renders P2 false. In Hawke's emerald case, if this liberal interpretation were adopted, a topaz would be qualified to play the role

27 Roca-Royes (2017). 
of counterpart. Given that topazes are not green, then the similarity principle allows us to justify the possibility of non-green emeralds. Thus, Hawke's argument for EI fails from the start.

Moreover, although it indeed expands the explanatory scope of the similarity principle if the counterpart relation is understood in a liberal sense, SET is nevertheless too strict to account for some possibility claims that we are entitled to believe. Let us consider (C3)-(C7). (C5)-(C6) are easy to justify if we take snow leopards, who are good at jumping, as similar counterparts of human beings. If we take a man, rather than a woman, as a counterpart of another woman, then (C7) can be justified. However, in the case of (C3) and (C4), given that nothing actually travels faster than light and nothing (on Earth) has a free-fall acceleration far more than $9.8 \mathrm{~m} / \mathrm{s}^{2}$, no matter what is taken as a counterpart of particles or apples, the possibility of (C3) and $(\mathrm{C} 4)$ cannot be justified via the similarity principle. Thus, the similarity principle is nevertheless too strict.

A Further Question: Might P2 be acceptable for another reason, different from the one that Hawke gives? ${ }^{28}$

My Response: This is a rather deep and difficult question, to which I can offer no definitive 'yes' or 'no.'

If one is able to defend $\mathrm{P} 2$, the modal epistemology invoked should be a satisfactory one, in the sense that it meets the following two requirements: first, it should not justify false possibility claims; second, it should be capable of accounting for all possibility claims that we are entitled to believe. ${ }^{29}$ Only if a satisfactory modal epistemology is available can we determine whether it can justify a given possibility claim, e.g., 'non-green emeralds are possible.'

However, as has been shown in Section II, CP, a form of modal rationalism, is not a satisfactory modal epistemology. It is either too liberal or too strict. ${ }^{30}$ SET faces the same problem. So, the approaches mentioned in this paper do not allow us to confidently answer whether P2 can be defended or not. Is there a chance in the future for us to give a confident answer? I tend to believe so. After all, many philosophers endeavour to offer a satisfactory modal epistemology. Crowford Elder, Stephen Biggs, and Roca-Royes are working in modal empiricism. ${ }^{31}$ Some philosophers, such as Carrie Jenkins, Peter Kung, and Tuomas Tahko, are even working towards providing a hybrid approach between modal rationalism and modal empiricism. ${ }^{32}$ But as to whether any of these approaches can defend P2, a separate discussion will be required.

\footnotetext{
28 I would like to thank an anonymous referee for raising this question.

29 A modal sceptic would add a third requirement, namely that this modal epistemology should not justify exotic possibility claims.

30 Roca-Royes also points out this problem. See Roca-Royes (2011).

31 See Elder (2005), Biggs (2011), and Roca-Royes (2017).

32 See Jenkins (2010), Kung (2010), and Tahko (2017).
} 


\section{Conclusion}

In this paper, I have argued that Hawke fails to defend EI. The reason is that SET, the only modal epistemology he accepts, is too strict to account for some justified possibility claims. Finally, we might re-consider why Hawke dismisses modal rationalism. He finds that modal rationalism faces a dilemma: the unconstrained CP is too liberal to establish some possibility claims that we are not entitled to believe; the conservative CP is too strict to account for some possibility claims that we are entitled to believe. However, as I have argued, modal empiricism faces the same dilemma: if SET incorporated more empirically informed methods, such as property recombination, then it would establish some possibility claims that we are not entitled to believe; if SET remains unmodified (even if we adopt a more liberal interpretation of the counterpart relation), it fails to account for some possibility claims that we are entitled to believe.

Acknowledgements: This paper is supported by the Ministry of Education of the People's Republic of China via the Humanities and Social Sciences Youth Fund (Project No. 19YJC720009). I am grateful to Asher Jiang (蒋运鹏), Ru Ye (叶茹), Xiaotao Liu (刘小涛), and the anonymous referees for their helpful comments and suggestions. I would also like to thank Sebastian Tants, Naomi Thurston, and graduate students at Wuhan University, such as Yi Yao (姚逸), Changqi Tian (田永奇), and Yao Niu (牛尧) for proofreading the text. I am deeply indebted to Dechao Su (苏德超) and Andreas Kemmerling for their mentorship during my time at Wuhan University and Heidelberg University.

\section{References}

Bealer, George

2002 "Modal Epistemology and the Rationalist Renaissance," in Conceivability and Possibility, edited by Tamer Gendler and John Hawthorne. Oxford: Oxford University Press, pp. 71-125.

Biggs, Stephen

2011 "Abduction and Modality." Philosophy and Phenomenological Research 83 (2): 283-326.

Bird, Alexander

2005 "The Dispositionalist Conception of Laws." Foundations of Science 10 (4): $353-370$.

Chalmers, David

2002 "Does Conceivability Entail Possibility?," in Conceivability and Possibility, edited by Tamer Gendler and John Hawthorne. Oxford: Oxford University Press, pp. 145-200.

Elder, Crowford

2005 Real Natures and Familiar Objects. Cambridge: MIT Press. 
Gendler, Tamar, and John Hawthorne

2002 "Introduction: Conceivability and Possibility," in Conceivability and Possibility, edited by Tamer Gendler and John Hawthorne. Oxford: Oxford University Press, pp. 1-70.

Hartl, Peter

2016 "Modal Scepticism, Yablo-Style Conceivability, and Analogical Reasoning." Synthese 193 (1): 269-291.

Hawke, Peter

2011 "Van Inwagen's Modal Skepticism." Philosophical Studies 153 (3): 351-364.

Hawke, Peter

2017 "Can Modal Skepticism Defeat Humean Skepticism?," in Modal Epistemology After Rationalism, edited by Bob Fischer and Felipe Leon. Cham: Springer, pp. 281-308.

Jenkins, Carrie

2010 “Concepts, Experience, and Modal Knowledge." Philosophical Perspectives 24 (1): 255-279.

Kung, Peter

2010 “Imagining as a Guide to Possibility." Philosophy and Phenomenological Research 81 (3): 620-663.

Prelević, Duško

2015 "Modal Empiricism and Knowledge of De Re Possibilities: A Critique of Roca-Royes' Account." Organon F 22 (4): 488-498.

Roca-Royes, Sonia

2011 “Conceivability and De Re Modal Knowledge.” Nous 45 (1): 22-49.

Roca-Royes, Sonia

2017 "Similarity and Possibility: An Epistemology of de re Modality for Concrete Entities," in Modal Epistemology After Rationalism, edited by Bob Fischer and Felipe Leon. Cham: Springer, pp. 221-245.

Shoemaker, Sydney

1998 "Causal and Metaphysical Necessity." Pacific Philosophical Quarterly 79 (1): 59-77.

Tahko, Tuomas

2017 "Empirically-Informed Modal Rationalism," in Modal Epistemology After Rationalism, edited by Bob Fischer and Felipe Leon. Cham: Springer, pp. 29-45.

Vaidya, Anand

2017 “The Epistemology of Modality," The Stanford Encyclopedia of Philosophy (Summer 2017 Edition), Edward N. Zalta (ed.), https://plato. stanford.edu/archives/sum2017/entries/modality-epistemology.

Van Inwagen, Peter

1998 “Modal Epistemology.” Philosophical Studies 92 (1-2): 67-84.

Yablo, Stephen

1993 "Is Conceivability a Guide to Possibility?" Philosophy and Phenomenological Research 53 (1): 1-42. 